\title{
Performance Issues in Shape Classification
}

\author{
Samson J. Timoner ${ }^{1}$, Pollina Golland ${ }^{1}$, Ron Kikinis ${ }^{2}$, Martha E. Shenton ${ }^{3}$, \\ W. Eric L. Grimson ${ }^{1}$, and William M. Wells III $^{1,2}$ \\ 1 MIT AI Laboratory, Cambridge MA, USA \\ \{samson, polina, welg, sw\}@ai.mit.edu \\ 2 Brigham and Women's Hospital, Harvard Medical School, Boston MA, USA \\ kikinis@bwh.harvard.edu \\ 3 Laboratory of Neuroscience, Clinical Neuroscience Division, Department of \\ Psychiatry, VAMC-Brockton, Harvard Medical School, Brockton, MA. \\ mshenton@warren.med.harvard.edu
}

\begin{abstract}
Shape comparisons of two groups of objects often have two goals: to create a classifier to separate the groups and to provide information that shows differences between classes. We examine issues that are important for shape analysis in a study comparing schizophrenic patients to normal subjects. For this study, non-linear classifiers provide large accuracy gains over linear ones. Using volume information directly in the classifier provides gains over a classifier that normalizes the data for volume. We compare two different representations of shape: displacement fields and distance maps. We show that the classifier based on displacement fields outperforms the one based on distance maps. We also show that displacement fields provide more information in visualizing shape differences than distance maps.
\end{abstract}

\section{Introduction}

Statistical studies of shape generally compare the shape of a structure selected from two different groups. They are used to form connections between shape and the presence or absence of disease 1,2, testing hypotheses in the differences between men and women [1,3/4, as well as examining biological processes. The goals of such studies are to classify new examples of a structure and to show a doctor the differences between classes.

This paper examines a set of issues in classifying structures and presenting information to doctors. Is it more important to use a good representation or a particular classification method? Is the choice of alignment technique critical? We examine these issues in one study: segmented amygdala-hippocampus complexes from fifteen normal and fifteen schizophrenic subjects [5].

\subsection{Classification Methods}

Until recently, most researchers classifying by shape used linear classifiers to separate two groups. The technology has generally been motivated by the desire to create deformable models of shape as the basis of automatic segmenters [6]. One creates a deformable model of shape using Principal Component Analysis

T. Dohi and R. Kikinis (Eds.): MICCAI 2002, LNCS 2488, pp. 355-362 2002.

(C) Springer-Verlag Berlin Heidelberg 2002 
(PCA) on representations of example structures. A generative model can then be made by allowing the shape to deform along the most important modes of variation. To compare two groups, one forms such a model for each group, and separates the two models using a hyperplane. Visualization of differences between classes can be examined by moving perpendicular to the hyperplane.

Golland et al. demonstrated that non-linear classification can potentially improve the separation between groups of shapes. The gradient of the classifier can be used to show differences between groups.

\subsection{Representation}

There are numerous attractive representations of shape for classification. Most representations implicitly determine the points on a surface. For example, surfaces can be parameterized in a series of spherical harmonics [1. Medial representations [7] are parameterizations of shapes based on a chain or sheet of atoms that project a surface. Distance maps embed a surface by labeling the voxels of a 3D-volume with the distance from the surface. Each of these parameterized models avoid establishing direct correspondences between surfaces.

Other representations, conversely, use explicit representations of correspondences. One can represent the surface of structures by a triangular mesh where the vertices of the mesh are at corresponding points on the different structures. One can also use volumetric displacement fields which establish correspondences on the surface as well as the interior of shapes.

Correspondence-based representations have the potential to yield more information than implicit representations. Displacement fields can show not only whether surfaces moved in or out, but can also show local rotation or compression of an organ. For this reason, we consider displacement fields in this paper.

Unfortunately, medical structures typically have large smooth surfaces so that finding correspondences is challenging. It is not intuitive where points in one smooth surface should lie on a second surface. One typically overcomes the challenge by matching two shapes while minimizing an additional constraint. For example, one can match structures by treating them as viscous fluids [8], though many have argued that this type of matching can form un-realistic correspondences. Finding corresponding surfaces that form a minimum description length of a dataset is a promising idea, though it is difficult to find in three spatial dimensions 9 . There have also been various types of surface matching by matching points of one surface to the closest points in another [1011].

Intuitively, in a good match, high curvature regions in one object match high curvature regions in a second object. Matching by minimizing an elastic energy should accomplish this feat. Matching a sharp portion of one surface against a sharp portion of another surface is lower energy than flattening the region to match against an less sharp adjacent region. Therefore, we align shapes using a linear elastic model.

\section{Methods}

We explore several issues that are important to shape-based classification. We examine two different representations of shape. We then compare the results of 
linear and non-linear classifiers. We explore the effects of normalizing the data by volume. Finally, we consider the effects of different alignment methods. Every combination is examined, though we report only a subset of the results.

The data consists of segmented amygdala-hippocampus complexes, fifteen from schizophrenic patients and fifteen from normal patients [5]. The objects are represented using both signed distance maps and displacement fields. Signed distance maps are formed by labeling each voxel with its distance from a surface $[2$. The resulting representation is the vector of labels of the voxels.

To form the displacement field representations of the left complexes, one left amygdala-hippocampus complex is chosen randomly as a basis. It is meshed with tetrahedra to facilitate the matching process. The mesh is then treated as a linear elastic material and deformed to match the amygdala hippocampus complexes as described in [12]. A similar procedure is carried out for the right complexes starting with meshing a complex and then matching to the rest of the data. For each match, the displacement of the nodes of the tetrahedra form a roughly uniform sampling of the displacement field. The resulting representation vector is the concatenation of all the displacements of the nodes of the tetrahedra.

When both sides are considered together, the vectors of each side are simply concatenated. Each section of the paper indicates whether data has been normalized by volume, or not scaled at all. Except for Section 3.3, all results use a second order moment alignment of the data. In that section, we also aligned using the second order moments of the mesh nodes, the mesh tetrahedra moments, and absolute orientation [13] (removing global translations and rotations from a deformation field). We tested aligning left and right amygdalas together and seperately.

Let $\boldsymbol{x}$ be the representation vector of the complexes for either representation. The squared distance between two amygdalas, $\left\|\boldsymbol{x}-\boldsymbol{x}^{\prime}\right\|^{2}$, is defined to be $(\boldsymbol{x}-$ $\left.\boldsymbol{x}^{\prime}\right)^{T}\left(\boldsymbol{x}-\boldsymbol{x}^{\prime}\right)$. For displacement fields, this distance is simply the square of the length of the displacement field between each complex. For distance maps, there is no simple interpretation of distance between shapes.

We train linear and non-linear classifiers of the data. The non-linear classifier is a support vector machine (SVM), described in [2]. We use the Radial Basis Function (RBF), $K\left(\boldsymbol{x}, \boldsymbol{x}_{k}\right)=-e^{\left\|\boldsymbol{x}-\boldsymbol{x}_{k}\right\|^{2} / \gamma}$, in the SVM where $\gamma$ is proportional to the square of the width of the kernel. We pick $\gamma$ to optimize the leave-one-out cross-validation accuracy of the classifier.

Our goal is not only to form the classifier, but to explicitly represent the shape differences between groups. Differentiating a pre-thresholded, SVM classifying function with respect to shape would seem to yield the answer. The derivative at $\boldsymbol{x}$ is $\sum_{k} \frac{2}{\gamma} \alpha_{k} y_{k}\left(\boldsymbol{x}_{\boldsymbol{k}}-\boldsymbol{x}\right) e^{-\left\|\boldsymbol{x}-\boldsymbol{x}_{k}\right\| / \gamma}$, where the $\left\{\alpha_{k}\right\}$ are constants determined by the SVM and $\left\{y_{k}\right\}$ are -1 for on group and 1 for the other. Using distance maps this answer is not sufficient. A small change to a distance map does not yield another distance map. Therefore, one must project a derivative back onto the manifold of distance maps [2]. However, displacement fields form a vector space; a small change in a displacement field yields another displacement field. Thus for this case, differentiating the classifier is sufficient for our goals. 


\section{Results}

We formed displacement fields between complexes using a linear elastic model [12]. Figure 1 shows a number of points found to correspond. The hand segmented complexes have notably different structure. The typical member of the data set has a nearly horizontal "tail" like the rightmost two complexes; a few have the tail at an angle (leftmost), or practically no such structure at all (second from the left). Even with the shape differences, in all examples, Point 1 stays slightly above the tip of the head and Point 2 stays on the side of the head. Examining the head of the complexes, some bases are nearly flat and level while others are curved and angled. A review of points near the base also shows that points are approximately in the same position relative to major structures.
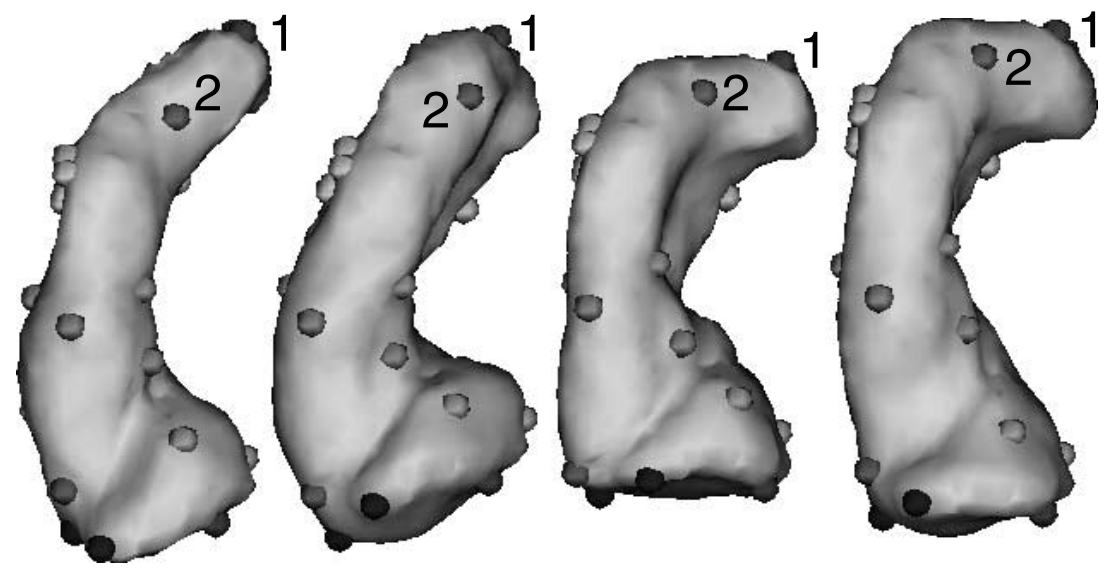

Fig. 1. Surfaces of four matched left complexes; the tail and head are at the top and bottom of the image respectively. To show correspondence across the shapes, points were randomly selected and represented by small spheres on each surface. Points 1 and 2 are referenced in the text.

\subsection{Classifier Comparison}

We compare the cross validation accuracy of linear and RBF-based 2 classifiers. Table 1 shows the classification accuracy using data normalized to remove relative volume. The table shows that support vector machines generally performed better than linear classifiers by 10 to 20 percentage points. We found this to be the case in all trials, with one exception. When deformation fields were aligned using absolute orientation [13], linear classifiers improved their classifying ability to as high as $70 \%$. (See Section 3.3.)

For individual sides, deformation field-based classifiers perform slightly better than distance map-based classifiers. When considering both sides together, that performance improvement becomes much larger. For comparison to other methods, Table 1] shows results from Gerig et al. [1] who made a classifier comparing 
Table 1. A comparison of cross-validation accuracy for RBF and linear classifiers. The data is normalized to the same volume. The range is the $95 \%$ confidence interval.

\begin{tabular}{|l||c|c|c|}
\hline \multirow{2}{*}{$\begin{array}{l}\text { Normalized } \\
\text { Structure }\end{array}$} & \multicolumn{3}{|c|}{ Cross Validation Accuracy using Linear Classifier } \\
\cline { 2 - 4 } & Displacement Field & Distance Map & \\
\hline Left Complex & $60 \pm 18 \%$ & $57 \pm 18 \%$ & \\
Right Complex & $53 \pm 18 \%$ & $53 \pm 18 \%$ & \\
Both Complexes & $57 \pm 18 \%$ & $53 \pm 18 \%$ & \\
\hline \hline Normalized & \multicolumn{2}{|c|}{ Cross Validation Accuracy using RBF } \\
\cline { 2 - 4 } Structure & Displacement Field & Distance Map & Gerig et al. \\
\hline Left Complex & $67 \pm 18 \%$ & $70 \pm 17 \%$ & \\
Right Complex & $73 \pm 17 \%$ & $77 \pm 17 \%$ & \\
Both Complexes & $80 \pm 16 \%$ & $67 \pm 18 \%$ & $73 \pm 17 \%$ \\
\hline
\end{tabular}

Table 2. Cross-validation accuracy for the different representations using RBF classifiers. For deformation fields and distance maps, the data is not normalized by volume. For the third column, volume was added separately to shape data [1].

\begin{tabular}{|l||c|c|c|}
\hline \multicolumn{1}{|l||}{ Structure } & \multicolumn{3}{c|}{ Cross Validation Accuracy using RBF } \\
\cline { 2 - 4 } & Displacement Field & Distance Map & Gerig et al. \\
\hline Left Complex & $77 \pm 17 \%$ & $73 \pm 17 \%$ & \\
Right Complex & $77 \pm 17 \%$ & $70 \pm 17 \%$ & \\
Both Complexes & $87 \pm 16 \%$ & $70 \pm 17 \%$ & $87 \pm 16 \%$ \\
\hline
\end{tabular}

the two sides in each subject using the same data. That classifier's accuracy is in between the two we tested.

\subsection{Including Volume}

Table 2 examines the effects of not normalizing for volume in the data, using RBFs. Comparing Tables [1] and [2] volume generates improvements for the displacement field based classifier of between 3 and 10 percentage points. For the classifier based on distance maps, volume improves or hurts a classifier, but the effect is roughly 3 percentage points each way. Gerig et al. [1] include volume as a separate feature in their classifier; doing so improves the performance of their classifier to the same as the deformation field-based classifier.

\subsection{Alignment}

We rigidly align shapes so that classifiers are not confused by variations in patient position during imaging. We tried the many alignment methods listed in Section 2 Choosing different alignment methods causes classifiers to have a range of between 3 and 10 percentage points, typically closer to $3 \%$. The worst results are shown in Table 1, $67 \%$ accuracy. The best results are $87 \%$ achieved in three different ways. Most results are between 70 and $80 \%$. Examining visualization of the differences between the classes as in Section 3.4, the different alignments had small impacts on the differences found between groups. 


\subsection{Visualization of Differences}

An important goal of this study is to visualize differences between the classes. Figure 2 shows those differences, found from derivatives of the classifier evaluated at the examples as described in Section 2 For displacement field based classifiers, we found the gradients to be visually similar evaluated at nearly all the left complexes, and visually similar at nearly all of the right complexes. For distance map based classifiers, the gradients were visually similar across right complexes, but not across left complexes. Figure 2 also shows that for both classifiers, the deformations detected for the two groups are similar in nature but opposite in sign.

In the bottom of Figure 2, the derivatives of the classifier based on deformation fields are shown in the form of a vector field (because distance maps do not use correspondences, it is not possible to show motions tangential to the surface using distance maps). The vector fields show that there is motion along the surface in several places. Most notably, there is a clear rotation of the "tail" of the complex in the image. Conversely, most of the motion in the base is simply compression or expansion. There is also a rotation in the head of the left amygdala, though much smaller in magnitude than the rotation in the tail, and very difficult to see in the image.

\section{Discussion}

We examined which issues in shape classification have the largest impact on classification accuracy. It is clear from Table 1 that the non-linear RBF-based methods outperform linear classifiers by 10 to 20 percentage points. Volume information (Table 22) consistently improves results the deformation field-based classifier, by 4 to 10 percentage points, as well as the classifier of Gerig et al. 1]. Thus, for this case, including volume information was helpful, but not as helpful as using a non-linear classifier over a linear one. Alignment techniques generally had a smaller effect. Differences between alignment methods generally produced accuracy changes of only a few percentage points.

In this study, displacement fields outperformed distance maps. Classification rates were higher in almost every test performed. Interestingly, Tables 1 and 2 suggest that deformation field-based classifiers were able to find correlations between the deformations on different sides to improve the classification rate, while distance maps-based classifiers were not. Perhaps most importantly, displacement fields provided vector fields in visualizations which added an important tool for visualizing shape differences.

One concern with these results is that the non-linear classifiers created from such small numbers of examples may not generalize to new data. That the classifiers found a qualitatively similar class difference evaluated at many complexes is a good indicator of those classifiers ability to generalize to other data. Also, the observation that in several cases, displacement-field based classifiers worked somewhat well with a simple linear classifier (Section 3.1) may also be such an indication. 


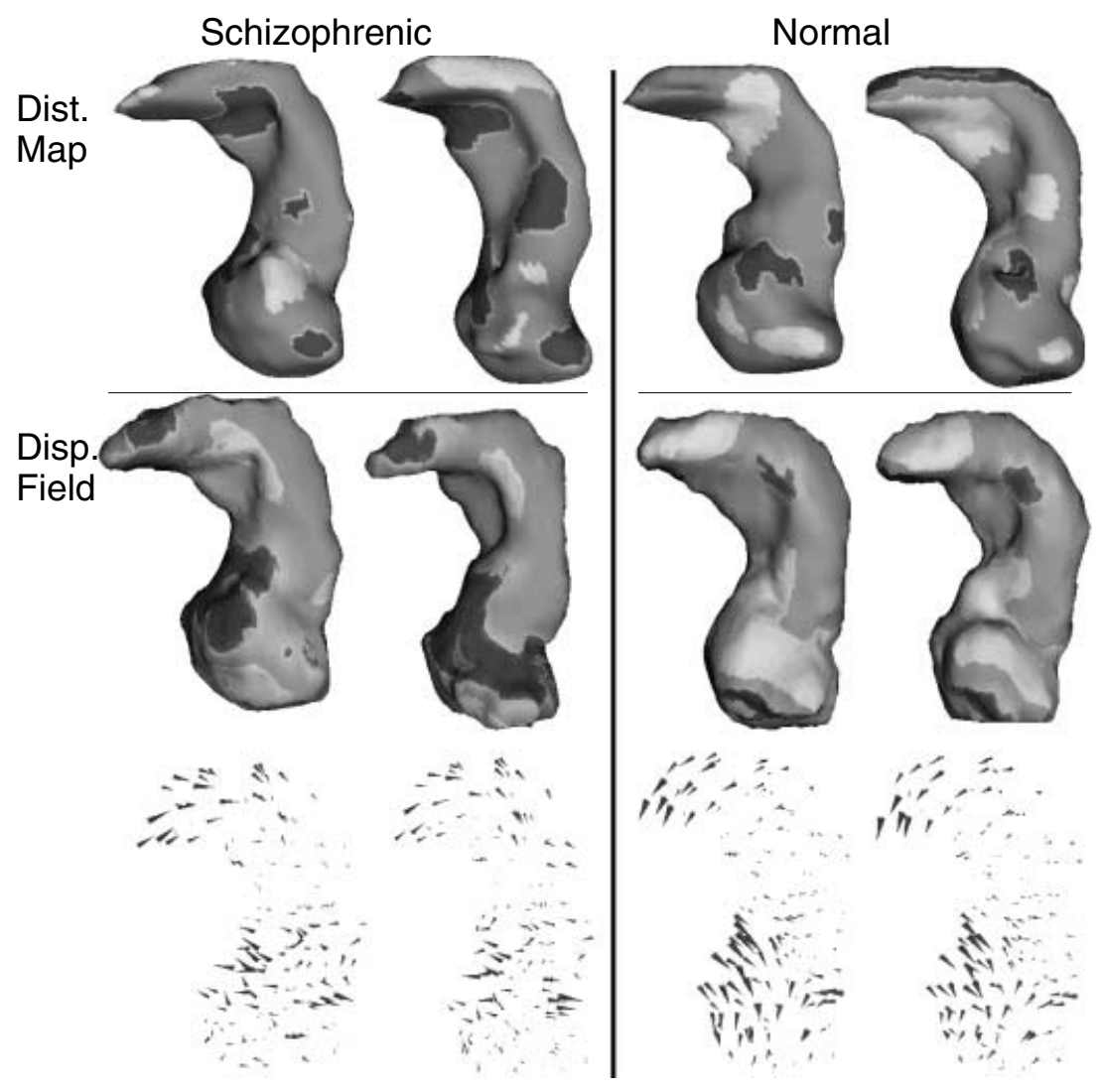

Fig. 2. The top four plots show the deformation of the surfaces relative to the surface normal for the left amygdala-hippocampus complex. For Schizophrenic subjects, "deformation" indicates changes to make the complexes more normal. For Normal subjects, "deformation" indicates changes to make the complexes more diseased. The 2x2 grid of surfaces shows deformations of Schizophrenic/Normal subjects using Distance Maps/Displacement Fields as representations. In each entry in the grid, the two largest deformations evaluated at the support vectors of the SVM classifier are shown; the larger one is on the left. The grayscale is used to indicate the direction and magnitude of the deformation, changing from white (inward) to grey (no motion) to black (outward). The bottom two plots are the deformations fields used to generate the plots directly above them. Note that motion along the surface does not affect the colors in the surfaces. For a color version of this figure, go to http://www.ai.mit.edu/people/samson/papers/publications.html

We are also concerned how well these conclusions generalize to other data sets. We believe that non-linear classification methods will almost always outperform linear classification methods. We also believe that the gains due to the inclusion of volume, or various alignment techniques exist, but will be much smaller. When correspondences can be found, correspondence-based methods do provide an advantage over non-correspondence-based methods because they provide additional information for visualizing class differences. 


\section{Conclusion}

We examined several issues that are important for performing shape comparison studies: complexity of the classifier, volume information, alignment method, and representation. For the shape differences between amygdala-hippocampus complexes, non-linear classifiers provide 10-20 percentage point accuracy gains over linear methods. For this study, not normalizing for volume provides a smaller gain, in the range of 4 to 10 percentage points. Using different alignment methods generally produce an even smaller impact on classification accuracy.

We have shown that for the cases examined, deformation field-based classifiers outperform distance maps as a measure of shape. Deformation fields form classifiers of higher accuracy and produce more information for the visualization of shape differences.

Acknowledgements: S.J. Timoner is supported by the Fannie and John Hertz Foundation and NSF ERC grant, J.H.U Agreement \#8810274. W. Wells is supported by the same NSF grant and NIH grant 1P41RR13218. P Golland is supported by NSF IIS 9610249 grant and a Martinos Center collaborative research grant. Dr. Shenton's data collection was supported by NIMH grants R01 50740 and K02 01110, and a Veterans Administration Merit Award.

\section{References}

1. G. Gerig, M. Styner, et al., "Shape versus size: Improved understanding of the morphology of brain structures," in MICCAI, (Utrecht), pp. 24-32, October 2001.

2. P. Golland, W. E. L. Grimson, M. E. Shenton, and R. Kikinis, "Deformation analysis for shape based classification," in IPMI, (Davis, CA), pp. 517-530, June 2001.

3. C. Davatzikos, et al., "A computerized approach for morphological analysis of the corpus callosum," Journal Computer Assisted Tomography, 20(1):88-97, 1996.

4. A. M. C. Machado and J. C. Gee, "Atlas warping for brain morphometry," in SPIE Medical Imaging, Image Processing, pp. 642-651, 1998.

5. M. E. Shenton et al., "Abnormalities in the left temporal lobe and thought disorder in schizophrenia: A quantitative magnetic resonance imaging study," New England Journal of Medicine, 327:604-612, 1992.

6. T. F. Cootes et al., "The use of active shape models for locating structures in medical images," Image and Vision Computing, 12(6):9-18, 1992.

7. S. Pizer et al., "Segmentation, registration and measurement of shape variation via image object shape," IEEE Trans. on Medical Imaging, 18(10):851-865, 1996.

8. G. Christensen, R. Rabbit, and M. Miller, "Deformable templates using large deformation kinematics," Transactions on Image Processing, 5(10):1435-1447, 1996.

9. R. H. Davies, T. F. Cootes, and C. J. Taylor, "A minimum description length approach to statistical shape modeling," in IPMI, (Davis), pp. 50-63, June 2001.

10. L. Cohen and I. Cohen, "Finite-element methods for active contour models and balloons for 2-d and 3-d images," PAMI, 15:1131-1147, November 1993.

11. L. Staib and J. Duncan, "Boundary finding with parametrically deformable models," PAMI, 14:1061-1075, November 1992.

12. S. J. Timoner, W. E. L. Grimson, R. Kikinis, and W. M. Wells, "Fast linear elastic matching without landmarks," in MICCAI, (Utretcht), pp. 1358-60, October 2001.

13. B. K. P. Horn, "Closed-form solution of absolute orientation using unit quaternions," Journal of the Optical Socienty of America A, vol. 4, pp. 629-642, 1987. 